\title{
REZAGO Y ABANDONO EDUCATIVO EN COLOMBIA: UN CAMBIO EN LA POLÍTICA PÚBLICA PARA LA EQUIDAD EDUCATIVA
}

\author{
SCHOOL DROPOUT AND LAG IN COLOMBIA. A PROPOSAL FOR PUBLIC POLICY \\ FOR EDUCATIONAL EQUITY
}

\author{
Ángela María Rojas Ladino ${ }^{1}$ \\ ${ }^{1}$ Facultad Latinoamericana de Ciencias Sociales. Maestría en Población y Desarrollo, México, e-mail: \\ angela.rojas@estudiante-flacso.mx
}

A R T I C L E I N F O
Article history:
Received 2018-09-06
Accepted 2019-06-17
Available online 2019-06-17
Palabras-clave: Equidad educativa. Rezago. Abandono. Desengache escolar. Política pública.

Keywords: Educational equity. Academic lag. School dropout. School disengagement. Public policy.

RESUMEN. A partir de la universalización de la educación básica, secundaria y media se han presentado nuevos retos en materia educativa, los estados latinoamericanos, entre ellos el colombiano, deben enfrentar el reto de garantizar acceso y equidad a la educación para toda la población mediante diversos instrumentos de política pública, esto se dificulta ante las diversas desigualdades que sufren nuestros países, diferencias sociales, económicas, culturales, de género que se ven reflejadas en desigualdades educativas y segregación de los entornos escolares. Así, procesos de largo alcance como el rezago y el abandono escolar, evidencian un proceso de desenganche del entorno escolar el cual está influenciado por factores familiares y escolares, tales como las estrategias de bienestar del hogar y el clima educativo de las instituciones escolares. A partir de este contexto se hace una revisión y propuesta de la política pública colombiana en torno a las problemáticas educativas alrededor del desenganche escolar, con el objetivo de proponer, desde una perspectiva institucional, una hipótesis de intervención que aborde la raíz causal de los factores escolares del rezago y el abandono escolar. Con lo anterior se propone una política encaminada a cambiar la relación que las y los jóvenes tienen con la escuela, basada en la amplitud de la jornada escolar y el abordaje de la idea de escuela democrática.

ABSTRACT. Since the universalization of various levels of education, new challenges had appeared. The Latin American states, including the Colombian, must face the challenge of ensuring access and equality in education for the entire population through different public policy instruments, this has been difficult due to the various social, economic, cultural, gender differences inequalities that our countries suffer, and these are reflected in educational inequalities and segregation of school environments. Thus, long-term processes such as academic lag and school dropout, evidence a process of disengagement from the school environment which is influenced by family and school factors, such as household welfare strategies and the educational climate of school institutions. Based on this, a review and proposal of the Colombian public policy regarding these educational problems is presented. From this context a review and proposal of the Colombian public policy is made around the educational problems around school disengagement, with the aim of proposing, from an institutional perspective, an intervention hypothesis that addresses the causal root of the school factors of dropout and academic lag. With the above, a policy aimed at changing the relationship that young people have with the school is proposed, based on the breadth of the school day and the approach of the idea of a democratic school. 


\section{Introducción}

Como en la mayoría de los países de América Latina, la educación en Colombia en todos sus niveles amplió su cobertura hacia finales del siglo XX y comienzos del XXI, cambios demográficos, económicos y sociales propiciaron su ampliación. Sin embargo, en la actualidad, la calidad de la educación que se imparte es muy baja en comparación con la de otras regiones del mundo (OCDE, 2016). Hoy en día se presentan diversas problemáticas en el sistema educativo formal, a pesar de que el país cuenta con altas tasas de asistencia escolar en educación básica, aún existe y persiste un porcentaje de población con rezago escolar o que ha abandonado la escuela, de esta manera la problemática se ha enfocado a identificar los factores intra y extra escolares que propician estos fenómenos, así como en el mejoramiento de la calidad.

Colombia se encuentra en una etapa plena de la transición demográfica, es decir, se ubica en el grupo de aquellos países con natalidad moderada y mortalidad moderada o baja, con un crecimiento natural moderado cercano al 2\% (CELADE; 1992). Los cambios en la estructura y dinámicas de la población mediante su crecimiento anual y los movimientos migratorios dentro y fuera del país se han visto reflejados en la implementación y conformación del sistema educativo colombiano. La importancia de la configuración del bono demográfico y con él una "ventana de oportunidades" para empezar un ciclo de beneficios y facilidades en las formas de vida de las sociedades, con altos estándares en los niveles de vida, implica grandes retos para las sociedades actuales, puesto que, como lo señalan Lee \& Manson (2003) no es suficiente con el incremento de la población para que se dé un incremento económico, sino que también es necesaria la implementación de políticas definidas por la estructura etaria de la población, entre las cuales entrarían las políticas educativas para todos los niveles. El reto de aprovechar el dividendo se complejiza en sociedades como la latinoamericana, en la cual, la transición demográfica es muy rápida, el nivel de ingreso es bajo y las instituciones son débiles. En este contexto, la educación toma gran importancia para el aprovechamiento del bono en el desarrollo de los países de la región, y sus formas de ejecución han sido variadas.

Después de la implementación de políticas de ajuste y reforma estructural que tienen cabida en la mayor parte de América Latina a partir de la década de los ochenta, las cuales producen el debilitamiento del sector público y junto con la ampliación de la obligatoriedad de niveles educativos, se genera un desbalance en el sector educativo en el que la escuela pública no tiene la capacidad de abarcar al total de la población. De esta forma, gran parte de la responsabilidad de la educación de la población es dada al mercado, a pesar de que garantizar el derecho a la educación es una función del Estado. En el caso de Colombia, esta función es dada al Estado como Estado pluriétnico y multicultural mediante la Constitución de 
1991. Esta hendidura creada en el sistema educativo entre responsabilidad estatal y funcionamiento privado empieza a abrir brechas de desigualdad educativas que se ligan a otras desigualdades de ingreso, de raza, de género, entre otras.

Recientemente, las políticas públicas en materia de educación han estado dirigidas a alcanzar metas en el acceso, calidad y equidad para la educación primaria y secundaria, las cuales se definen en seis puntos del Plan Nacional de Desarrollo 2010-2014. Pero un primer paso para alcanzar las metas propuestas es aumentar el gasto público en educación, el cual es muy bajo en comparación con otros países de América Latina (el gasto del país oscila entre el $4 \%$ y el $6 \%$ del PIB mientras que el de otros países de la región está alcanza el 7\% (OCDE, 2016)). Este bajo gasto se ve reflejado en los niveles de la calidad educativa del país, como se puede ver en los resultados de las pruebas PISA (2012) los cuales denotan bajos puntajes en el país, incluso entre los latinoamericanos.

Las condiciones de equidad de la educación también influyen en su calidad. La equidad en los resultados educativos también se ve reflejada en los resultados promedio del aprendizaje, según las pruebas PISA, la equidad en la educación se define al proporcionar a todos los estudiantes, independientemente de su sexo, antecedentes familiares o situación socioeconómica, oportunidades similares para beneficiarse de la educación (PISA \& OCDE, 2013), bajo este parámetro, Colombia se encuentra por debajo del promedio del desempeño en matemáticas y por debajo del promedio de equidad en los resultados educativos.

A pesar del aumento en la cobertura, las condiciones de pobreza siguen siendo un obstáculo para acceder y permanecer en un sistema educativo de calidad. Según Sarmiento (2010) la población mayor de 5 años del decil más pobre tiene en promedio una cuarta parte de los años de escolaridad de la población en el decil más rico (2,9 frente a 12,9 años) (Sarmiento, 2010 en Delgado, 2014)). De esta forma, la falta de equidad ha ido produciendo un fenómeno de segregación escolar en donde la estratificación socioeconómica de las escuelas y colegios contribuye a las desigualdades educativas en el país. Esto se puede ver en los resultados de las pruebas SABER 5, 9 y 11 que se implementan a estudiantes de estos grados de secundaria y media para medir saberes y competencias que deben aprender en dichos niveles, según la OCDE (PISA \& OCDE, 2013) se evidencia que hay una gran diferencia en el rendimiento académico entre los estudiantes de escuelas públicas y privadas, independiente del puesto y de las competencias evaluadas. Estas diferencias también se ven entre los estudiantes de educación pública en las zonas urbanas y rurales. Así, el nivel de la calidad educativa de los colegios públicos es significativamente menor que la de los colegios privados además de la zona donde se encuentre.

Estas inequidades se agudizan en zonas de conflicto. Colombia ha vivido un conflicto armado durante las últimas décadas que ha afectado a gran parte de la población, especialmente en las zonas rurales del país. El conflicto armado impacta substancialmente a los niños y jóvenes, ya que fenómenos como el desplazamiento forzado, el reclutamiento de 
menores, la agudización de la pobreza, entre otros, son obstáculos para que los jóvenes continúen con normalidad procesos sociales como cursar una trayectoria escolar continua y acceder posteriormente al campo laboral de manera exitosa, de esta forma las oportunidades educativas y laborales en zonas de conflicto se vuelven limitadas. Si bien en los últimos años se ha entrado en una etapa de posconflicto a partir de procesos de paz con los distintos actores armados, aún siguen persistiendo hechos de violencia (física, política y estructural) en las zonas marginadas del país.

Bajo este contexto, los retos para los niveles de educación secundaria y media aún son grandes, los problemas de repitencia, abandono y rezago son persistentes, y en los distintos niveles del sistema educativo colombiano se presentan problemas en la cobertura, permanencia y calidad de la educación, los cuales se agudizan según las características socioeconómicas de los estudiantes. Esta brecha de segregación educativa genera desigualdad en los resultados educativos de los jóvenes, en lo que debería ser un derecho desde un principio de equidad para todas y todos los ciudadanos.

A partir de esta problemática, el objetivo del presente trabajo es estudiar cómo procesos de truncamiento escolar como el rezago y el abandono en la educación secundaria y media en Colombia son producto de un proceso de desenganche escolar, el cual está relacionado con factores familiares y escolares, que propicia una violación al derecho de la educación para un sector de la población, generando desigualdades educativas que se ligan a su vez a otras desigualdades estructurales, a partir de esto, se realiza una breve revisión de la política pública en la materia y una propuesta basada en una hipótesis de intervención.

Para esto se operacionalizan los conceptos de rezago y abandono escolar utilizando la Encuesta de Calidad de Vida de Colombia del DANE del año 2016. Ambos fenómenos son vistos como procesos de largo alcance y a partir de su análisis se hace un breve recorrido por las principales políticas públicas que se han implementado recientemente en el país (sin pretender realizar una evaluación de política o un reporte de sus resultados). Finalmente, se proponen acciones de política pública para una raíz causal en específico: los factores escolares.

\section{Los enfoques de igualdad de oportunidades en la educación}

Los cambios en el sistema educativo han dependido de distintas visiones de igualdad y acceso, el principal enfoque que ha tenido el derecho a la educación es desde la perspectiva de igualdad de oportunidades, este enfoque es propuesto a partir del paradigma utilitarista de la igualdad, en el cual, siguiendo las ideas de Smith de actor racional y el principio maximizador, se genera una idea de igualdad que se ve traducida en el sistema educativo en igualdad de acceso a las instituciones de educación. De esta forma, durante el siglo XX en 
América Latina, la implementación del derecho a la educación se dio mediante la universalización del acceso principalmente a los niveles de básica y secundaria, basándose en ideales de universalización y de homogenización, planteando criterios escolares desde una igualdad de oportunidades meritocrática, en esta perspectiva "únicamente el esfuerzo personal y voluntario, fruto de la libre elección, justificaría la desigualdad de resultados" (Bolívar; 2012;15).

Pero la evidencia empírica ha mostrado que la igualdad utilitarista por sí sola no evita que se reproduzcan desigualdades en el sistema educativo, en América Latina ha sido evidente la relación entre desigualdad en el ingreso y rendimiento promedio de los estudiantes (Cuenca, 2012). De esta forma, empiezan a ser tomados en cuenta otros aspectos de la vida social que explican los rendimientos educativos de los estudiantes, importa el contexto e importa el origen social, asimismo, importan las identidades y las diferencias. Es así como se adopta un paradigma de equidad, si bien los conceptos de equidad e igualdad están correlacionados, no deben confundirse, puesto que implican distintos juicios éticos y de justicia social (Sánchez-Santamaría, J., \& Ballester Vila, M. G; 2013), desde la perspectiva de equidad educativa que considera a los sujetos como objetos de justicia y se establece el derecho a la educación garantizada por los Estados.

De esta manera, se empieza a cuestionar qué produce y reproduce la escuela como institución social. Con las ideas de Amartya Sen, se amplía el marco conceptual de justicia social tomando un enfoque de capacidades, se extiende el debate sobre la igualdad al entenderse en un contexto más amplio en el que se tienen en cuenta un gran conjunto de demandas sociales. Las desigualdades se pueden dar en distintos ámbitos: en los ingresos, las libertades, los derechos, etc. Desde esta perspectiva, la educación debe brindarles a los individuos las capacidades necesarias para alcanzar un alto grado de bienestar y para ser ciudadanos con funcionamientos básicos similares. La mera inclusión no es suficiente, puesto que también se discrimina contra los que son incluidos en condiciones de desigualdad, es lo que Amartya Sen llama la "inclusión desfavorable" o la "exclusión de la inclusión equitativa", es por esto que la educación debe tener presente la diversidad cultural (Hevia; 2010).

A partir de esta distinción, la OCDE (2016) plantea que la equidad educativa debe articularse en torno a las dimensiones de inclusión y justicia social. Por un lado, la inclusión debe considerar el acceso, la participación y los logros de los alumnos, prestando atención a las diferencias de origen, a aquellos en riesgo de estar excluidos y marginados (UNESCO, en Sánchez-Santamaría, J., \& Ballester Vila, M. G; 2013). Por otro lado, la justicia social se debe encaminar a garantizar para todas y todos el acceso y el alcance al aprendizaje de saberes que permitan realizar sus proyectos de vida, desde una perspectiva de "capability", de dar acceso a la educación para que cada quien pueda desarrollar sus capacidades personales.

Al complejizar los conceptos de inclusión y equidad también se complejizan las políticas educativas, se deben tener en cuenta tanto las políticas compensatorias y de 
discriminación positiva como la políticas preventivas y sistémicas destinadas a evitar la exclusión (Echeita; 2010), teniendo presente la importancia de la calidad de la educación que se imparte. Desde su antónimo, la exclusión educativa no se ve solamente como el aislamiento total del sistema, sino como un proceso de progresivo alejamiento, con elementos como la precariedad y la acumulación de barreras que impiden el acceso a mecanismos de protección (Laparra et al., 2007 en Ramírez; 2016). De esta manera, los fenómenos educativos de rezago, sobreedad y abandono hacen parte de este tipo de exclusión.

Más allá de la perspectiva de capacidades, que suele ser individualista, la perspectiva de inclusión educativa toma en cuenta tanto los factores intraescolares como los extraescolares y, alejándose de la idea de homogenización, las diferencias se asumen como constantes (Ibíd.). Esta visión impulsa propuestas como la de educación para la democracia, en la cual las capacidades que crean los estudiantes también están dirigidas a la participación activa en las entidades escolares, tomando en cuenta a la familia y al entorno. Desde la Declaración de Salamanca y su Marco de Acción de la Unesco en 1994 las acciones dirigidas a la educación dan un giro hacia el planteamiento sistemático de la inclusión educativa (UNESCO, 1994). Asimismo, en América Latina, se han realizado distintos proyectos sobre esta línea encaminados a fortalecer la participación democrática dentro de la escuela que implican activamente a toda la comunidad educativa (Elboj, Puigdellivol, Soler, y Valls, 2002; Nilhom, 2006 en Ramírez; 2016).

De esta forma, las distintas visiones de la igualdad de oportunidades (como libertad natural, como meritocracia, como igualitario o universal, o como compensatoria), así como las acciones que se han tomado según la ideología (como el incentivo a la educación privada y la competencia o las acciones compensatorias y la discriminación positiva), van cambiando la forma de implementar la política pública educativa a través del tiempo.

Durante la década de los 90, América Latina apropia una seria de políticas de tipo compensatorio, las cuales son políticas territorializadas y paternalistas para luchar contra las desigualdades educativas, pero estas medidas mostraron ser insuficientes para democratizar la enseñanza, puesto que ignoran lo que pasa en el interior del sistema escolar, en cómo funciona y se configura el currículum como generador de desigualdades. Posteriormente, las políticas se basan en la lucha contra la exclusión y la cohesión social, las cuales se enfocan en que la ciudadanía tenga garantizado el acceso a un nivel básico de bienes sociales (educación, oportunidades, salud) y se sientan incluidos en la sociedad, pero sin profundizar en abogar por una sociedad igualitaria. $Y$ finalmente a partir de los años 2000 , aquellas basadas en la individualización y maximización de oportunidades de éxito, donde se plantea la "escuela inclusiva" que implica mirar al interior de la escuela para adaptarla a la diversidad de los estudiantes y su contexto y así alcanzar las potencialidades de cada uno (Bolívar, 2012). 


\subsection{La educación en Colombia}

Para establecer el panorama general del sistema educativo en Colombia en la actualidad e identificar las razones por las cuales se producen procesos de alejamiento del entorno escolar que puede manifestarse como rezago y abandono se utilizó la encuesta de hogares de tipo transversal ECV-2016 (Encuesta de Calidad de Vida de Colombia del año 2016 realizada por el DANE). En la figura 1 se pueden observar tres tasas representativas escolares: la tasa de asistencia escolar (TAE), que para el nivel de secundaria es de $93 \%$ y para media baja a $79.09 \%$; la tasa de cobertura neta (TCB) que se presenta en ambos casos sobre el $100 \%$, lo que indica que hay más personas en edades diferentes a la normativa en ambos niveles; y la tasa de cobertura neta (TCN) que se restringe sólo a la población en edad normativa para cada nivel, y se presenta con $80,71 \%$ para secundaria y baja hasta el $57,80 \%$ para media, es decir, que casi la mitad de los jóvenes entre 16 y 17 años no están asistiendo al nivel educativo medio. La diferencia entre las dos tasas de cobertura evidencia el rezago, mientras que la tasa de asistencia muestra el abandono.

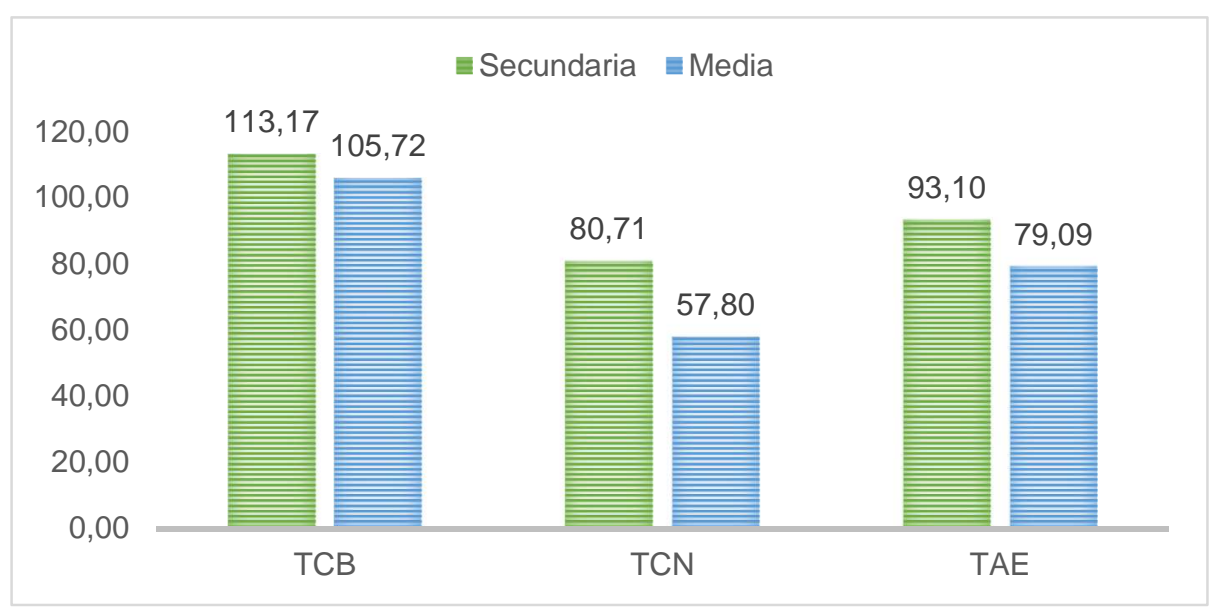

Figura 1 - Tasas escolares, educación secundaria y media, Colombia, 2016

Fuente: Elaboración propia a partir de la ECV-2016 (2018)

En cuanto a los fenómenos de truncamiento escolar, los datos de la encuesta muestran que (ver cuadro 1) del total personas del grupo etario de 12 a 17 años que estudian (edades normativas para secundaria y media), el 17,81\% está rezaga del nivel, es decir, están en un nivel menor del que deberían estar según su edad normativa, si desagregamos el rezago por grado, es decir, si el alumno cuenta con rezago de un año o más en el grado en que se encuentra, aumenta la proporción a un $38.5 \%$. En ambos casos, la mayoría de estudiantes que presentan rezago son hombres. 


\section{Cuadro 1 - Población rezagada por nivel, grado y sexo. Educación secundaria y media. Colombia 2016.}

\begin{tabular}{|l|r|r|r|r|}
\hline \multicolumn{2}{|c|}{ Población rezagada por nivel } & \multicolumn{2}{c|}{ Población rezagada por grado } \\
\hline & \multicolumn{1}{c|}{ Freq. } & \multicolumn{1}{c|}{$\%$} & Freq. & \multicolumn{1}{c|}{$\%$} \\
\hline Hombre & $3,703,293$ & 82.19 & $2,771,303$ & 61.51 \\
\hline Mujer & $802,230.78$ & 17.81 & $1,734,220$ & 38.49 \\
\hline Total & $4,505,524$ & 100 & $4,505,524$ & 100 \\
\hline
\end{tabular}

Fuente: Elaboración propia a partir de la ECV 2016 (2018)

Para el año 2016, el 9,4\% de jóvenes del grupo etario de 12 a 17 años abandonaron la escuela o no estaban asistiendo a ninguna institución educativa al momento de la encuesta, de los cuales el $62 \%$ son hombres y el $38 \%$ son mujeres. La razón que más manifestaron las y los jóvenes es "no le gusta o no le interesa el estudio" con un $40 \%$, seguida por "otras razones" (de carácter individual como enfermedades), la "falta de dinero, costos de estudio elevados o necesita trabajar", y "por situaciones académicas y escolares" (que incluye: inseguridad o malos tratos en el establecimiento educativo, falta de cupos, no existe centro educativo cercano o el establecimiento asignado es muy lejano; bajos resultados en el periodo académico y repetición de cursos), finalmente el encargarse de los oficios del hogar y por embarazo constituyen, cada una, el $15 \%$ de los casos. Estas dos últimas razones declaradas son casi exclusivamente femeninas, mientras que "necesita trabajar" es sobre todo una razón masculina. Estas dinámicas de abandono muestran, por un lado, cómo se van afianzando los roles de géneros y los papeles que cumplen las y los jóvenes en la producción del bienestar del hogar, a medida que va aumentando la edad, los hombres se ven más expuestos a la búsqueda de empleo y las mujeres hacia razones de cuidado y encargarse de las labores del hogar. $Y$ por otro, las primeras razones nos remiten a la configuración interna del currículum y el sistema educativo, a una no identificación de las y los jóvenes con el mismo.

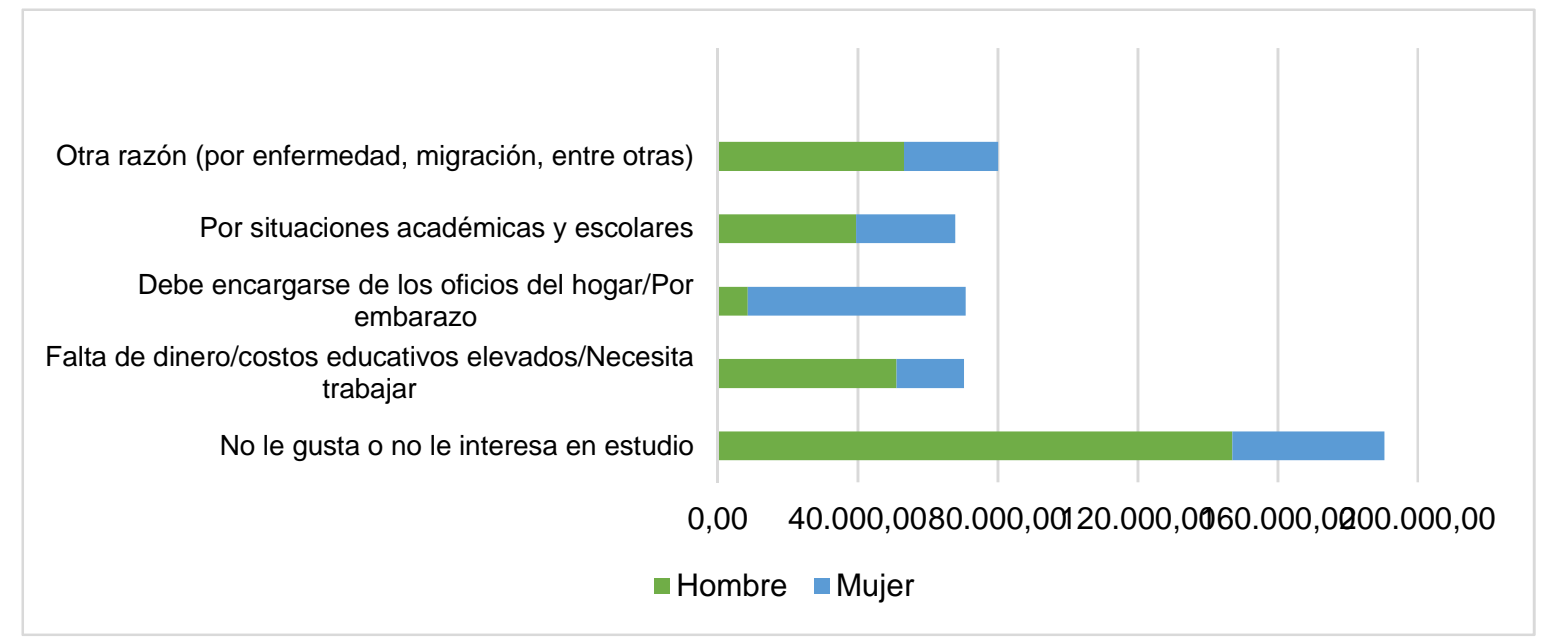

Figura 2 - Razones autodeclaradas por las cuales no estudia la población de 12 a 17 años. Colombia 2016.

Fuente: Elaboración propia a partir de la ECV-2016 (2018) 
De esta forma, los factores escolares y familiares (como las estrategias de trabajo productivo y de trabajo de cuidado en los hogares, las relaciones y el clima educativo de las instituciones educativas, entre otros) inciden en el desarrollo de las trayectorias de los alumnos, los cuales pueden iniciar un proceso de desenganche escolar que toma en cuenta el valor que se le da a la educación así como su calidad (no me gusta a escuela, no me sirve para nada) y que finalmente contribuyen a la decisión de alejarse o abandonar la escuela. (Rumberger, 2001 en Gonzáles; 2006).

\section{Análisis de la política pública en Colombia}

La intensidad y la urgencia de la política educativa se da por la importancia que esta tiene en la vida de las personas y en el desarrollo de las sociedades, el paso a la vida a la vida adulta y a la vida laboral está en gran parte determinado por el paso por la escuela; las trayectorias escolares y las trayectorias laborales determinan la vida de las personas de tal forma que la manera en la que se desarrollen influirá en la economía del país, la ciudadanía, la democracia, entre otros elementos claves para las naciones, por estos motivos, los problemas relacionados con la educación han estado constantemente presentes en la agenda pública de los gobiernos. Desde una perspectiva racional, la cual se basa en un modelo hipotético-deductivo se propone el siguiente árbol de problemas para entender las raíces del problema a tratar.

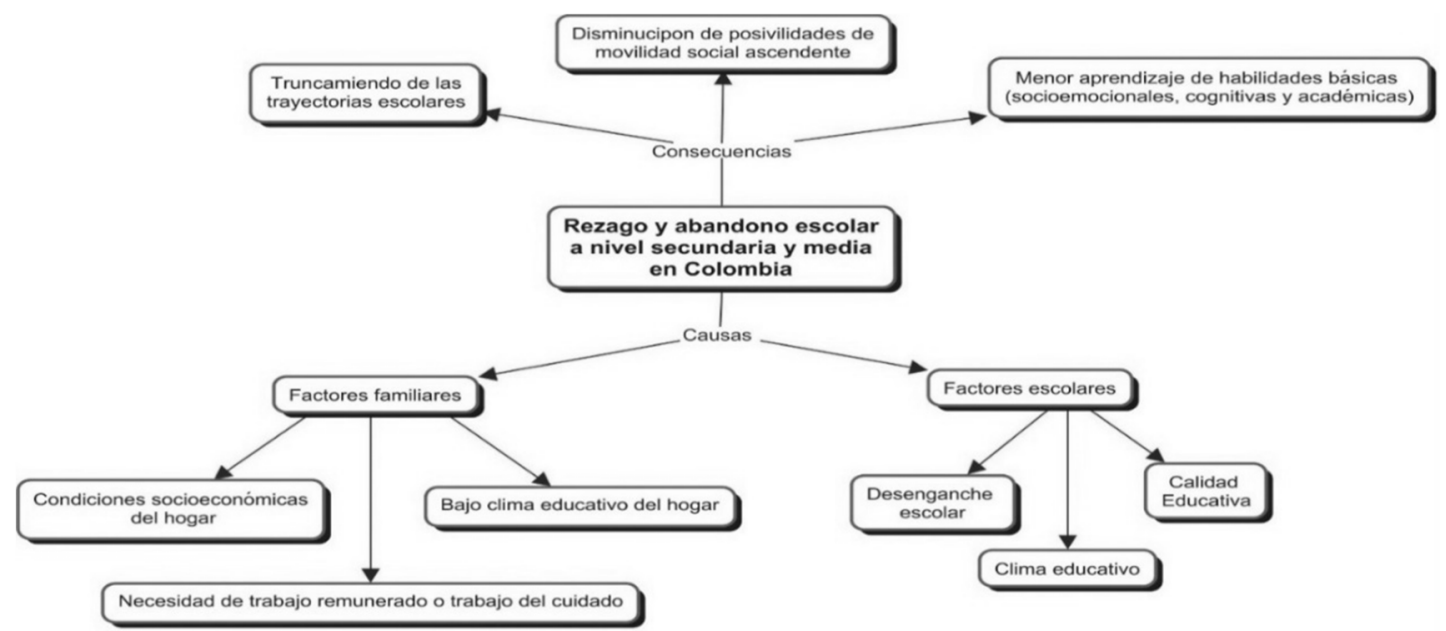

Figura 3 - Árbol de problemas

Las causas que se visibilizan de los problemas de rezago y abandono escolar a nivel secundaria y media en Colombia son de dos tipos: causas a partir de factores familiares y causas a partir de factores escolares, es importante recordar que esta es una división analítica y que, en la realidad, estos dos factores se interrelacionan en el espacio de la comunidad a la que pertenecen los estudiantes, sus familias, la comunidad académica, y en el contexto del sistema educativo del país. Entre las causas familiares encontramos las condiciones socioeconómicas del hogar, relacionado a esto se encuentra la necesidad de trabajo 
remunerado o trabajo de cuidado y el clima educativo del hogar que relaciona variables como los años de escolaridad de los padres o del jefe(a) de hogar, el promedio de años de escolaridad del hogar, los hábitos de lectura en el hogar, entre otros.

Por otro lado, entre los factores escolares se toman en cuenta tres elementos, la calidad educativa de la institución, la cual puede ser medida por los resultados en pruebas de saberes y habilidades como las pruebas PISA o las pruebas SABER; el clima educativo, entendido como un conjunto de significados, expectativas y valores que los miembros de la escuela comparten respecto de sus tareas, sus relaciones y su entorno, teniendo en cuenta dimensiones de interacción cultural, grupal y motivacional, las cuales brindan significados compartidos sobre el sentido de la educación (Blanco; 2007 en PNUD-SED, 2015; 199); y finalmente, relacionado con los dos elementos anteriores, el desenganche escolar, como un proceso en el cual se toman en cuenta los atributos, valores, actitudes y conductas del estudiante que contribuyen a la decisión de abandonar la escuela. (Rumberger, 2001 en Gonzáles; 2006).

A partir de este planteamiento se escogió la raíz causal de los factores escolares, y más específicamente el desenganche escolar como raíz causal de la intervención, puesto que los factores escolares son más inmediatos, el acotamiento del problema a un espacio concreto permite enfocar los esfuerzos a soluciones específicas, alejándose de la visión de la cual el origen socioeconómico es el único causante del fracaso escolar, se establece el peso de la responsabilidad a la escuela como institución socializadora formadora de ciudadanía y democracia para todas y todos. Sin embargo, no se debe olvidar que el mayor involucramiento de la familia y del entorno en las acciones propuestas generará un impacto mayor en los resultados. Con esta perspectiva institucional se propone el siguiente triángulo de actores para exponer cómo se abordará la raíz causal seleccionada.

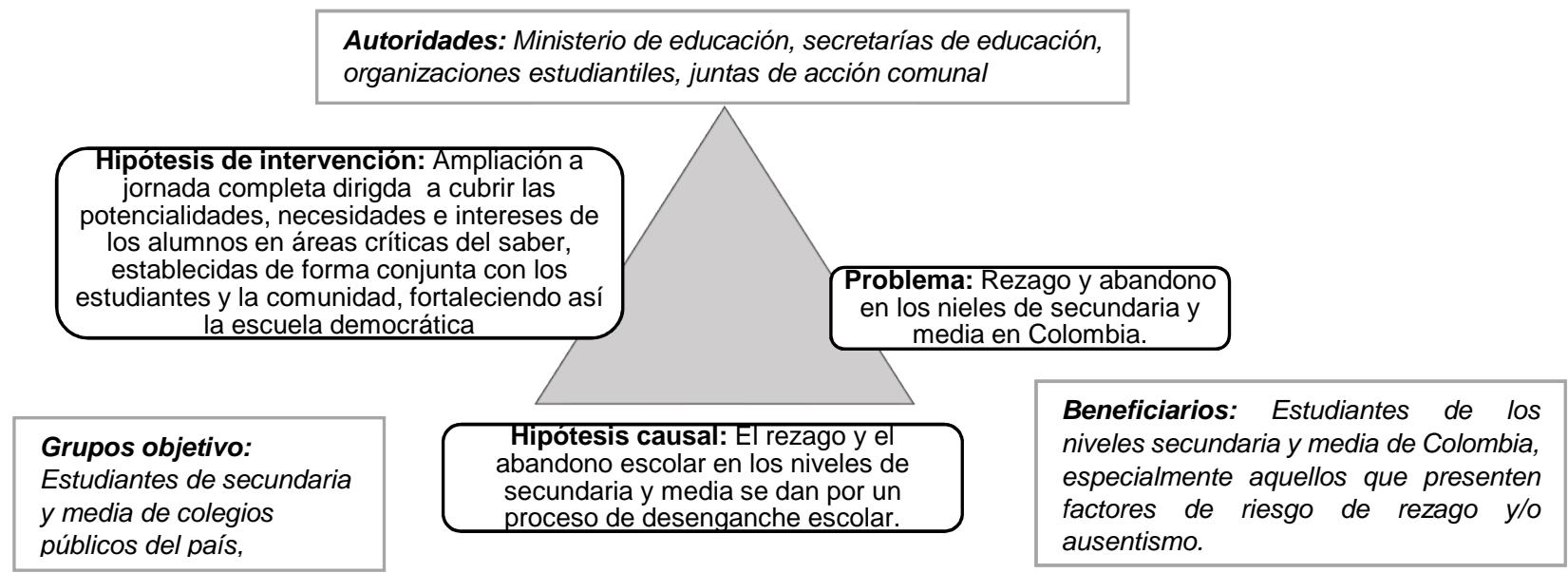

Figura 4 - Triángulo de actores

Fuente: Elaboración propia (2018) 
Una de las formas de observar el desenganche escolar de las y los jóvenes es mediante las razones autodeclaras de abandono, recordemos que la mayoría dice que abandona el sistema educativo porque no le gusta o no le interesa el estudio, este desinterés finalmente resulta en trayectorias escolares truncadas. No hay que olvidar que tanto el rezago como el abandono son procesos de largo alcance, dados a lo largo de los años escolares y que se agudiza en el nivel secundaria (como lo muestran las tasas ya mencionadas).

La hipótesis de intervención propuesta pretende frenar las conductas propias del proceso de desenganche escolar, con dos medidas realizables al interior de la escuela. Primero, mediante la ampliación de la jornada escolar, y segundo, mediante el fortalecimiento de la escuela democrática, por medio de la participación de los alumnos y si es posible de las familias, en distintas áreas y prácticas. El objetivo de estas dos medidas es mejorar la relación de los estudiantes con la escuela, haciendo la oferta educativa significativa para los alumnos y mejorando el clima escolar. Con esta hipótesis de intervención se pretende que baje el número de estudiantes que se rezagan y abandonan las instituciones educativas en estos niveles puesto que se crea un lazo más fuerte con la institución, con los otros compañeros, con la comunidad y con la idea misma de escolaridad. Esto es importante en un contexto de posconflicto como el colombiano, puesto que la desvalorización de la educación como ámbito de desarrollo de las capacidades puede llevar a los jóvenes a perpetuar conductas delictivas y reproducir ideas de intolerancia, afiliación a grupos armados, al narcotráfico, entre otras actividades propias del estado de conflicto. Colombia debe permitir y asegurar acceder y permanecer a la escuela a todos los jóvenes que en otro momento optaron por la guerra, brindando en ésta un lugar de oportunidades, de educación de calidad, democrático y participativo, restituyendo el derecho a la educación como responsabilidad estatal.

\subsection{Instrumentos de política pública y recomendaciones}

Las políticas públicas orientadas a problemas educativos han puesto en marcha numerosos instrumentos, algunos han sido exitosos en contextos específicos, otros han logrado transferirse a otros escenarios y otros no han tenido muy buenos resultados. En el caso de Colombia actualmente las prioridades de la política educativa están enfocadas al acceso y el mejoramiento de la calidad académica, específicamente, con la implementación del Plan Decenal de Educación se han efectuado distintos programas y proyectos, y a futuro se espera que se implementen más programas integrales y que se profundice en los ya existentes para la mejora de la educación, especialmente en zonas rurales y la atención a jóvenes desplazados o afectados por la guerra.

Una de las herramientas que se ha implementado en mayor medida son los programas de transferencia condicionadas (como "Familias en Acción"), estos programas han tenido resultados positivos en muchos contextos, principalmente en el aumento de matrícula en los 
primeros años, pero su eficacia se va perdiendo a medida que los jóvenes van creciendo, así que no es la política correcta para la raíz causal seleccionada.

También se han instaurado en el país programas de beca/premio, tales como "Ser pilo paga" el cual brinda incentivos a los alumnos para que permanezcan en la escuela secundaria y becas universitarias en universidades privadas a los alumnos con mejor promedio en las pruebas SABER 11, este programa está dirigido a incentivar a los alumnos de menores recursos para mejorar su rendimiento escolar y continuar al nivel de educación superior. Este tipo de acciones dirigidas a los mejores alumnos se enmarca en una visión meritocrática y conservadora de la igualdad de oportunidades, dejando de lado el capital social y cultural de los alumnos y de sus entornos, destacando a los pocos que pueden salir del mismo. La escuela no sólo debe ser el espacio que premia a los estudiantes sobresalientes de situaciones adversas, sino que debe brindarle a todos los alumnos que están en estas situaciones las oportunidades para desarrollar el capital social y cultural que muchas veces no encuentran en el hogar, de esta forma se estaría garantizando un modelo de escuela con equidad e inclusión.

Por otro lado, los programas basados en la enseñanza y el acompañamiento que se dan en el aula de clase han dado buenos resultados. Existen estrategias que van desde el universalismo, las cuales se desarrollan en el conjunto del centro escolar y van dirigidas a todo el alumnado (acciones como servicios a los alumnos y sus familias, intervenciones tempranas dirigidas a solventar problemas de asistencias, entre otros), hasta aquellas más focalizadas, que se centran específicamente en los alumnos que presentan factores de riesgo (Gonzáles, 2014), tal es el caso del programa "La escuela busca al niño".

Entre el primer grupo de políticas (universalistas) está la ampliación de la jornada, la cual le permite a las alumnas(os) ahondar en distintos tipos de conocimientos y a la familia reducir el tiempo de cuidado, pero como señala Gómez (2014) no es suficiente con que las alumnas(os) estén físicamente dentro de un salón de clases, sino que participen activamente de la vida social y académica de la institución, un tipo de absentismo también es no participar en ninguna actividad académica, lo que remite, a su vez, a la calidad y pertinencia del currículo, a la enseñanza y la institución educativa como factores que lo prevengan. De esta forma, la ampliación de la jornada no debe plantearse simplemente como el número de horas que los alumnos pasan en la escuela, sino cómo invierten ese tiempo. Un ejemplo de esto es el programa "40x40" implementado en los colegios públicos de la ciudad de Bogotá, en el que en ampliación de la jornada se enseñan temas como: matemáticas, pensamiento tecnológico, desarrollo de robótica; expresión oral y escrita; aprendizaje de una segunda lengua; reflexión y análisis sobre ciencias sociales y ciencias naturales. Así como áreas de desarrollo integral, donde se desarrollan capacidades de ciudadanía y convivencia (PNUD-SED; 2015). 
Siguiendo con esta línea, están los programas que buscan replantear un modelo de escuela democrática basada en la inclusión, tal es el caso de distintos proyectos en América Latina con este enfoque y con resultados satisfactorios. Uno de ellos es el proyecto de participación comunitaria como estrategia para abordar el trabajo infantil y la falta de oportunidades implementado en Chile, cuyo objetivo es crear una comunidad de aprendizaje a partir de la construcción de alianzas entre diversos agentes; de igual forma, está el proyecto de escuela como espacio para la transformación educativa, en Querétaro, México, cuyo propósito es buscar alternativas a los problemas del desencanto escolar expresado en situaciones de fracaso y deserción. $Y$ finalmente, el proyecto de modelos escolares para la equidad, en varias ciudades de Colombia, el cual pretende elevar las condiciones de acceso, retención y permanencia en la escuela, así como los resultados de aprendizaje. Todos estos proyectos estuvieron encaminados a la búsqueda de la inclusión social, se llevaron a cabo en entornos empobrecidos y de marginación social y lograron mejorar los resultados educativos (Ramírez; 2016). Así, la idea de democratización de la escuela pública tiene el objetivo de democratizar esta institución y la gestión local de la educación mediante vínculos de colaboración para enfrentar desigualdades en los aprendizajes y así mejorar problemas de desenganche escolar que finalmente se puedan ver reflejados en rezago o abandono.

Existen políticas desde enfoques preventivos hasta enfoques más paliativos, así, se propone una política encaminada hacia un enfoque preventivo, que evite que los jóvenes empiecen a experimenten un proceso de desenganche escolar. La propuesta de intervención pretende combinar dos tipos de política pública que han resultado exitosas, por un lado, la universalización de la jornada única, y por otro, el fomento de la escuela democrática. Se espera que los alumnos, y en la medida de lo posible las familias, se vean involucradas en la toma de decisiones sobre el currículo y sobre los enfoques y saberes que se implementarán en la ampliación de la jornada, de esta forma se tomarán en cuenta tanto los factores institucionales como contextuales propios de cada escuela, territorializando la misma. Dejando de lado políticas con enfoques meritocráticos que sólo se enfocan en un porcentaje pequeño de alumnos sobresalientes pero que no garantizan el éxito educativo y la participación activa y democrática al interior de la escuela para todas y todos los jóvenes. Para que este enfoque sea exitoso, es importante un compromiso de los distintos niveles de gobierno para brindar las condiciones de todo tipo, tanto materiales y de infraestructura, como de capital humano, de docentes con condiciones laborales dignas y procesos evaluativos que tengan en cuenta el contexto escolar.

\section{Consideraciones finales}

Los problemas educativos han estado en la agenda pública de los gobiernos de la región durante décadas, si bien en un primer momento los esfuerzos se encaminaron a la 
universalización y el acceso a los niveles de educación básica y secundaria, a partir del gran aumento en la matrícula, la discusión se ha dirigido a problemas de calidad y de segregación de la educación, la cual es producto de desigualdades sociales que se reproducen en el ámbito escolar y que se han agudizado por el contexto de violencia y desigualdad económica propio de la realidad colombiana actual, superar estas desigualdades ha sido el reto en materia de política pública.

Tanto el abandono como el rezago son fenómenos de largo alcance que hacen parte de un proceso de desenganche escolar, de falta de significación de la escuela por parte de los alumnos que finalmente abandonan del todo los estudios, actualmente la mayoría de jóvenes que abandona la escuela lo hace porque perdieron el interés en ella. La propuesta de intervención para esta problemática se deberá centrar en un enfoque preventivo, el cual fortalezca los lazos entre los alumnos y la escuela, esto implica intervenir elementos intrínsecos al funcionamiento de la escuela y el currículum como la jornada escolar, el clima escolar y la participación de los alumnos y la comunidad al interior de la misma.

Si bien se han implementado numerosas iniciativas de política pública para intervenir los problemas educativos, que van desde programas de transferencia condicionadas hasta escuelas democráticas, se plantea una intervención que implique dos acciones: la ampliación de la jornada y la ampliación de los espacios de toma de decisiones y debate de los alumnos, los docentes y la comunidad en cuanto al currículo. Esto crearía un sentido de pertenencia a la escuela, puesto que se llenarían de significado las líneas de estudio, desde lo que los estudiantes, los docentes y las familias crean que es el conocimiento que necesitan los alumnos de acuerdo a su entorno y al territorio; y finalmente, se estimularía la participación democrática por parte de los jóvenes. Este punto de resignificación del papel de la escuela toma importancia en un contexto de posconflicto y proceso de paz del país, en el cual se deben encontrar maneras en que la comunidad encuentre nuevas formas de diálogo y resolución de conflictos más allá de recurrir a acciones violentas y actores armados, brindando herramientas democráticas desde los primeros espacios de socialización como lo es la escuela, en su interacción con el entorno, las familias y los problemas comunales.

Este artículo presenta de forma propositiva una propuesta a una raíz causal específica, la cual propone un enfoque de equidad e igualdad de oportunidades, diferente al enfoque meritocrático predominante actualmente. Sin embargo, es necesario ahondar en evaluaciones de las políticas propuestas que ya se han puesto en marcha, así como en estudiar las condiciones para su implementación efectiva, tales como las condiciones laborales de los docentes, las instalaciones, la infraestructura, la cohesión social de la comunidad, el papel que juega la escuela dentro de la misma, entre otras. 


\section{REFERENCIAS}

BOLIVAR, A. Justicia social y equidad escolar. Una revisión actual. Revista Internacional de Educación Para La Justicia Social (RIEJS), 1(1), 9-45, 2012. Disponible en: http://www.rinace.net/riejs/numeros/vol1-num1/art1.pdf

CELADE. La transición demográfica en América Latina. Centro Latinoamericano y Caribeño de Demografía, 1992. Disponible en: https://www.cepal.org/celade/sitdem/de_sitdemtransdemdoc00e.html

CUENVA, R. Sobre justicia social y su relación con la educación en tiempos de desigualdad. Revista Internacional de Educación Para La Justicia Social, 1(1), 79-93, 2012.

DELGADO, M. La educación básica y media en Colombia: retos en equidad y calidad. Los Desafíos de Educación Preescolar, Básica Y Media En América Latina, 123, 2014.

ECHEITA, G. Inclusión y exclusión educativa. De nuevo “voz y quebranto”. Revista lberoamericana sobre Calidad, Eficacia y Cambio en Educación, 11(2), 19-29, 2010. Disponible en: http://www.redalyc.org/pdf/551/55127024005.pdf

GONZÁLEZ, M. T. Absentismo y abandono escolar: Una situación singular de la excusión educativa. REICE: Revista Electrónica Iberoamericana Sobre Calidad, Eficacia Y Cambio En Educación, 4(1), 1-15, 2006.

HEVIA, R. El Derecho a la Educación y la Educación en Derechos Humanos en el contexto internacional. Revista Latinoamericana de Educación Inclusiva, 4, 25-39, 2010.

SÁNCHEZ-SANTAMARÍA, J., \& BALLESTER VILA, M. G. (2013). Desarrollando el éxito educativo para todos: reflexiones, propuestas y retos conceptuales en torno a la equidad educativa. Revista Iberoamericana Sobre Calidad, Eficacia Y Cambio En Educación, 12(2), 85-104.

LEE, R. \& MASON, A. What is the demographic dividend? Bekerly University. Pp. 1-11, 2003.

MINISTERIO DE EDUCACIÓN NACIONAL. Educación: visión 2019. Ministerio de Educación MEN, 2109, 62, 2006.

OCDE. Educación en Colombia. Aspectos Destacados, 16, 2016. Disponible en: http://www.plandecenal.edu.co/cms/media/herramientas/oecd_educacion_en_colombia_aspectos.pdf

PISA \& OCDE. Results: Excellence Through Equity (Vol. II), 2013. Disponible en: https://doi.org/10.1787/9789264201132-en

PNUD, P. de N. U. para el SED. Educación de Calidad para una ciudad y un país equitativos. Bogotá, Colombia, 2015.

RAMÍREZ, A. Repensar la inclusión social desde la educación: algunas experiencias en América Latina. Revista Internacional de Educación Para La Justicia Social, 5(1), 177-194, 2016. Disponible en: https://doi.org/10.15366/riejs2016.5.1 